\title{
Capturing implementation knowledge: applying focused ethnography to study how implementers generate and manage knowledge in the scale-up of obesity prevention programs
}

Kathleen P. Conte ${ }^{1,2^{*}}$ (D) Abeera Shahid ${ }^{3}$, Sisse Grøn ${ }^{4}$, Victoria Loblay ${ }^{4}$, Amanda Green ${ }^{5}$, Christine Innes-Hughes ${ }^{5}$, Andrew Milat ${ }^{6}$, Lina Persson ${ }^{6}$, Mandy Williams', Sarah Thackway ${ }^{6}$, Jo Mitchell ${ }^{8}$ and Penelope Hawe ${ }^{4}$

\begin{abstract}
Background: Bespoke electronic information management systems are being used for large-scale implementation delivery of population health programs. They record sites reached, coordinate activity, and track target achievement. However, many systems have been abandoned or failed to integrate into practice. We investigated the unusual endurance of an electronic information management system that has supported the successful statewide implementation of two evidence-based childhood obesity prevention programs for over 5 years. Upwards of $80 \%$ of implementation targets are being achieved.

Methods: We undertook co-designed partnership research with policymakers, practitioners, and IT designers. Our working hypothesis was that the science of getting evidence-based programs into practice rests on an in-depth understanding of the role programs play in the ongoing system of local relationships and multiple accountabilities. We conducted a 12-month multisite ethnography of 14 implementation teams, including their use of an electronic information management system, the Population Health Information Management System (PHIMS).

Results: All teams used PHIMS, but also drew on additional informal tools and technologies to manage, curate, and store critical information for implementation. We identified six functions these tools performed: (1) relationship management, (2) monitoring progress towards target achievement, (3) guiding and troubleshooting PHIMS use, (4) supporting teamwork, (5) evaluation, and (6) recording extra work at sites not related to program implementation. Informal tools enabled practitioners to create locally derived implementation knowledge and provided a conduit between knowledge generation and entry into PHIMS.

\footnotetext{
* Correspondence: kathleen.conte@sydney.edu.au

${ }^{1}$ The Australian Prevention Partnership Centre, Ultimo, NSW 2007, Australia ${ }^{2}$ Menzies Centre for Health Policy, School of Public Health, and University Centre for Rural Health, Faculty of Medicine and Health, The University of Sydney, Sydney, New South Wales 2006, Australia

Full list of author information is available at the end of the article
} 
(Continued from previous page)

Conclusions: Implementation involves knowing and formalizing what to do, as well as how to do it. Our ethnography revealed the importance of hitherto uncharted knowledge about how practitioners develop implementation knowledge about how to do implementation locally, within the context of scaling up. Harnessing this knowledge for local use required adaptive and flexible systems which were enabled by informal tools and technologies. The use of informal tools also complemented and supported PHIMS use suggesting that both informal and standardized systems are required to support coordinated, large-scale implementation. While the content of the supplementary knowledge required to deliver the program was specific to context, functions like managing relationships with sites and helping others in the team may be applicable elsewhere.

\section{Contributions to the literature}

- In clinical medicine, mundane tasks hidden from formal recording processes are thought to underlie the success or failure of efforts to get evidence-based programs into practice.

- In population-level prevention, we show that part of the success of a state-of-the-art electronic information management system accompanying statewide scale-up may be the unreported and extra work undertaken by practitioners and captured in supplementary recording technologies.

- "Informal" recording technologies demonstrate that practitioners generate additional knowledge about implementation in the course of practice as they accommodate local accountabilities alongside "top-down" goals.

- Nimble technologies are needed to respond to emerging knowledge produced by self-organizing systems at scale

\section{Introduction}

The scale-up of effective programs no longer relies simply on passive methods of education or knowledge diffusion. Instead, the focus is on developing purposeful strategies and mechanisms to mobilize the translation and enactment of research knowledge into practice on a large scale. In Australia, a landmark health information technology (IT) system-the Population Health Information Management System (PHIMS) - is currently supporting the largest-ever implementation of evidence-based childhood obesity prevention programs in the country. Funded by the New South Wales (NSW) government, the Healthy Children Initiative (HCI) is a suite of evidence-based policies and programs to address childhood obesity through settings-based approaches [1].

PHIMS supports the large-scale implementation of two of HCI's flagship programs-Live Life Well @ School and Munch and Move ${ }^{\odot}$. Both programs support the implementation of evidence-based practice recommendations from the World Health Organization including that schools and childcare settings create healthy food environments, and that these settings have adequate facilities to support physical activity [2]. Live Life Well @ School targets primary school settings while Munch and Move ${ }^{\oplus}$ targets early childhood services. Both programs are effective in producing settings-based environmental changes that promote healthy eating and physical activity practices $[3,4]$. They are achieving high reach at scale with $89 \%$ (3348/ $3766)$ of childcare services and $81 \%(2133 / 2566)$ of primary schools participating [5]. Ongoing monitoring has shown steady progress in the implementation of practices over time. Over the period of 2012-2015, the proportion of early childhood services serving only water or age-appropriate drinks increased from 33\% to $71 \%$ [6]. In schools, physical activity at breaks, teacher learning, and development for healthy eating and physical activity and other practices likewise significantly increased [7].

Via a four-way practitioner-policymaker-IT developer-researcher partnership, we had the opportunity to study PHIMS use, and through PHIMS, the implementation of HCI. Because HCI has been delivered and sustained since 2011, we were particularly interested in how information about program implementation has been used and managed for sustained implementation, supported, in part, by the PHIMS system.

There is increasing recognition that understanding how implementers use, adapt, and importantly, create new knowledge in practice is key to understanding why some implementation endeavors are successful while others fail. In clinical settings, previous research has exposed the importance of "hidden work" (i.e., largely unseen work) completed by practitioners that is required to adopt, integrate, and sustain an innovation in practice [8]. The seemingly mundane tasks that often go unnoticed or unrecognized likely underlie the success of implementation endeavors. We set out to understand the extent to which this may also be the case in the delivery of population-level prevention programs. To our understanding, our study is the first of its type. There is little documentation of IT systems in 
population-health contexts, and what does exist suggests that these systems fail more often than succeed [9]. To our knowledge, PHIMS is rare in that it enjoys sustained use and was recently expanded to include health issues beyond obesity. Studying PHIMS in-depth provides an opportunity to observe the knowledge gathered, used, and generated by practitioners in the day-to-day implementation of a scaled-up program. It is a unique opportunity to explore any unseen work that exists alongside PHIMS use. Insights will be valuable for IT design and uncovering previously undiscerned dynamics of implementation. Specifically, this study aims to examine how practitioners use and create knowledge in the ongoing implementation of obesity prevention programs. Our research objectives were (1) to explore what kinds of information and knowledge are valued by practitioners for implementation and (2) to examine how PHIMS sits alongside other systems used by practitioners to generate and capture such knowledge for implementation.

\section{Using PHIMS to track program implementation}

HCI is delivered by 14 teams of Health Promotion Officers (hereafter referred to as "practitioners") situated across 15 local health districts (LHDs) funded and supported through the NSW Ministry of Health [5]. Annual health service agreements [10] set out service and performance expectations to ensure the provision of safe, high-quality, patient-centered healthcare services including prevention. Collectively, HCI teams support over 6000 primary schools and early childcare services to achieve a specified number of evidence-based practices as described above and elsewhere [11]. The achievement of these practices constitutes the key performance indicators (KPIs) by which implementation progress and fidelity are monitored and measured. PHIMS was developed to capture this performance data and was designed with a dual purpose: to (1) support the implementation of HCI programs while (2) simultaneously aggregating and reporting data for implementation monitoring and to inform future policy and programmatic decisions.

The HCI performance monitoring approach [11] includes two levels of indicators for assessment: Service Delivery Indicators-i.e., reach, follow-up, and support provided by practitioners-and KPIs-i.e., specified implementation targets for $\mathrm{HCI}$. These two functions are built into the design of PHIMS itself and enable PHIMS to simultaneously serve multiple key user groups:
- Health Promotion Officers: enter data about their day-to-day work and use PHIMS to support the delivery of the programs. They generate the primary data that PHIMS aggregates and reports to other users.

- Managers of HCI teams: monitor achievement of program practices and the activities and impact of their team members.

- The Office of Preventive Health: monitor program implementation, enabling statewide responses to specific targets which need strengthening or quality improvement. These users can access aggregated data for the LHD in real-time. They are unable, however, to access site-level data and notes.

- The Ministry of Health: track the LHD's delivery on KPI targets. Reports generated from PHIMS data are used in performance meetings with LHD Chief Executives.

The development of PHIMS and a detailed description was previously reported [12]. Briefly, PHIMS is protected through a series of access control settings that are configured according to the users' role. Figure 1 provides an overview of PHIMS' main functions. Users at the practitioner-level are provided an overview of their district's performance compared against statewide performance. Users access their assigned sites via three main interfaces:

1) General information about each site, e.g., contact persons and training status of staff.

2) Questionnaires about how the sites are performing on implementation targets. Users enter data via a Likert-type survey form. PHIMS aggregates the data and feeds it back via user reports.

3) System-generated reports about training, sites requiring follow-up, practice achievement, or program adoption.

\section{Methods}

This study is part of a larger project, co-produced through a collaboration between researchers, policylevel decision-makers in NSW health, IT designers of PHIMS, and state-level HCI coordinators. The research approach and specific research questions were designed over a period of extensive collaboration between the partners and with the overarching aim of examining how PHIMS intersects with $\mathrm{HCI}$ practice [13]. This co-production process continued between the larger research team and partners throughout the currently reported project. The current article uses data from ethnographic field notes collected with all 14 HCI teams in New South 


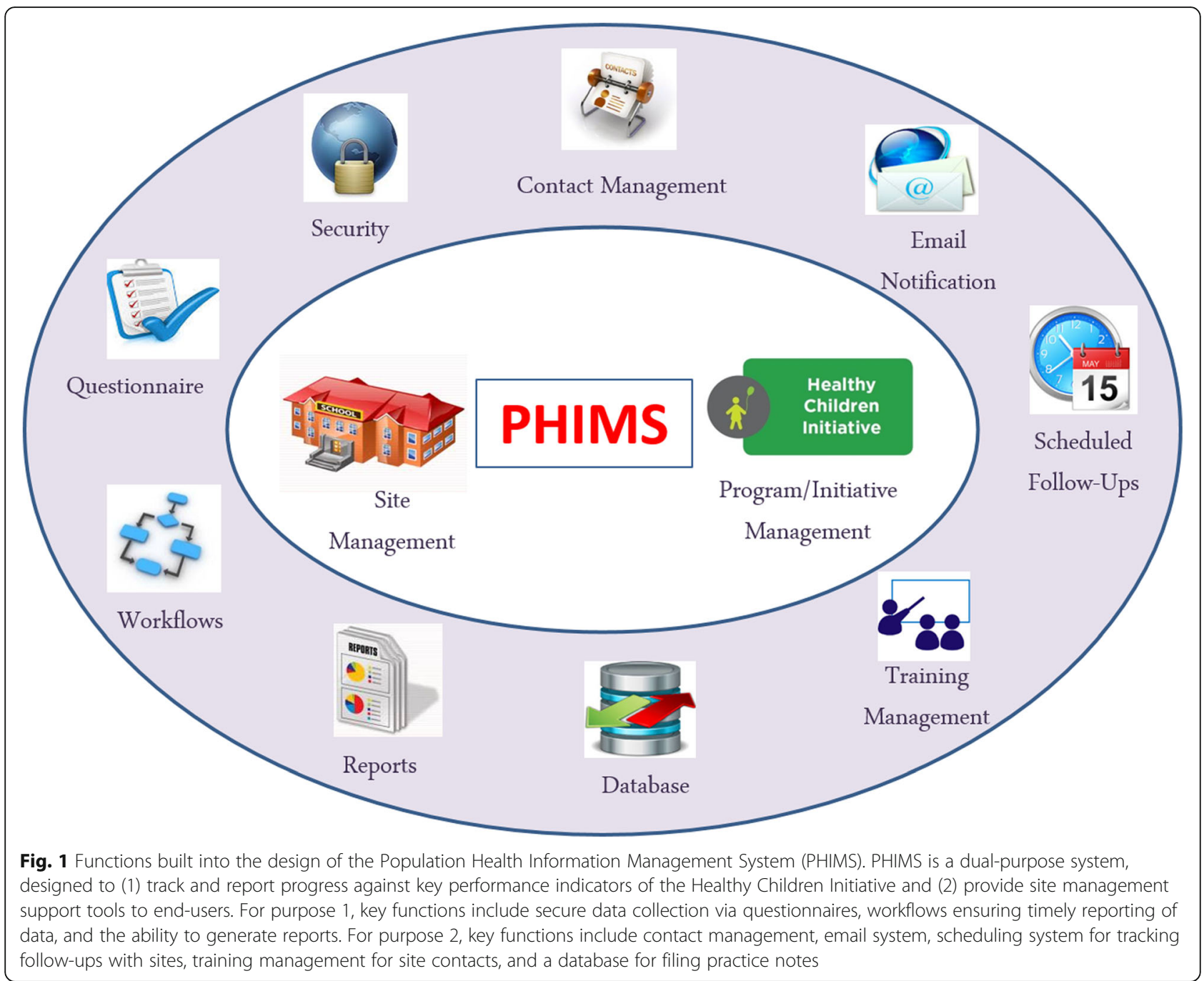

Wales. ${ }^{1}$ We used the consolidated criteria for reporting qualitative research (COREQ) guidelines [14] to guide our study and referred to the Standards for Reporting Qualitative Research (SRQR) [15] reporting (for more details, see Additional file 1).

The research was informed by the concept of multisited ethnography, in which the "field" is grounded in systems rather than geographic locations [16-18]. In this way, we conceptualized the field as a system of connections between people, places, and objects mediated through and by PHIMS [17]. In each local health district, we adopted an approach consistent with "focused" ethnography [19], characterized by intensive data collection during site visits with practitioners but where contact with sites preceded and extended beyond in-person visits (detailed below). We drew on a range of theories (see [13] for extended list) as sensitizing concepts prior to

\footnotetext{
${ }^{1}$ Note: There were 15 LHDs in New South Wales at the time of the study. One team delivered HCI programs in two LHDs.
}

entering the field to "suggest directions along which to look" [20], and throughout the writing, analysis, and interpretation process. Incorporating theory throughout all aspects of the project reflects an ongoing dialog between theory and practice [21] and enabled us to recognize specific constructs during data collection and initial analysis that related to our guiding research questions while remaining open to emergence.

\section{Data collection}

Three researchers (KC, SG, and VL) undertook ethnographic fieldwork across a period of 12 months (August 2016-2017). One (KC) conducted preparatory work for 1 year prior to entering the field in which she conducted exploratory interviews with project partners, undertook training in PHIMS, and met with multiple sites to design the fieldwork approach with $\mathrm{PH}$. Consistent with a focused ethnographic approach [19], the team of ethnographers immersed themselves in this data, undertook a 3day intensive collaborative site visit, and engaged in 
extensive theoretical readings prior to independently entering the field. Each independent field visit was followed by a group debrief and reflexive discussions of the fieldwork experiences.

We shadowed, interviewed, or observed 106 practitioners across all 14 teams. Access to teams and participants varied depending on trust built, the participants' willingness to host researchers, and interest in the topic. Geography also influenced access in that ethnographers traveling to teams in regional areas spent intensive periods varying from 1 to 5 days. For some of the metrobased sites in closer proximity, researchers were able to selectively schedule visits around activities and meetings over a longer period.

What our focused approach to ethnography lacked in terms of duration in the field it made up for in purposeful selection and engagement with participants and a variety of data sources [21]. The focused nature of visits resulted in intensive engagements where ethnographers worked alongside participants in their offices and accompanied them when they visited schools, workshops, and meetings. Fieldwork activities were documented in extensive field notes providing us with a large quantity of data for the time spent on visits [19]_over 500 pages of typed, single-spaced field notes in the dataset. Other data collected-pre-, during, and post-fieldwork-includes photos (about 65), written program documents and materials (over 100 documents), ad hoc recorded interviews (over $50 \mathrm{~h}$ have been transcribed verbatim), and subsequent email discussions with participants (numerous and ongoing). Following site visits, continuous iterations of fieldwork included presenting at statewide health promotion workshops, holding statewide video webinars, returning to LHDs for feedback workshops, and giving seminars to the PHIMS IT design team. As is typical of focused ethnography methods, these engagements enabled researchers to reengage with the ethnographic context long after leaving the field, each time gaining new insights and allowing flexibility to explore emerging issues [21]. This approach differs from other short-duration ethnography types-often called "rapid ethnographies" now widely adopted in computer studies and increasing in healthcare studies [22] - and is typically applied to inform specific practice changes.

The ethnographic research team also consulted and met with the broader co-production partnership team (the authors of this paper) periodically throughout the fieldwork to discuss select, anonymized results from our dataset. In this way, the co-production partnership group acted as "para-ethnographers" whose reflexive awareness of the ethnographic material helped to co-inform our research process [23, 24]. The continuous circulation and reinterpretation of data with research participants and our partners extended the ethnographic "place" beyond the intensive field trips to include processes of data analysis and dissemination [21]. These sharing processes also facilitated reflexive practice among the ethnographers, prompting ongoing consideration of our role, perspectives on the data, and our ability to facilitate change through the research process [25].

\section{Analysis}

The research question guiding this analysis was established with the project partners at the outset of the research project. Other papers in our project (e.g., Loblay V, Conte K, Groen S, Green A, Innes-Hughes C, Milat A, et al: Collaborative friction and knowledge generation: a coproduction dialogue within a researcher-policymakerpractitioner partnership examines the value of unreported practice. In preparation) explicitly draw on multiple data sources and adopt a more explicitly reflexive approach to analysis akin to traditional ethnographic approaches. For this analysis, however, we adopted a focused approach to systematically code field note data to answer the guiding research question. Therefore, this analysis is consistent with conventional content analysis [26] from which subsequent codes were developed via a grounded approach. As described fully in our protocol [13], we used NVIVO [27] to develop an overall project codebook to organize and sort data at a high level. Using this codebook, we extracted content from material coded as "tools and methods other than PHIMS that are used to organize, monitor, and structure practice." This resulted in 142 instances of coded content (ranging from a sentence to multiple paragraphs) from 44 unique field notes. First, we identified the range and type of specific tools or methods used in addition to PHIMS. Next, we identified 24 keywords, mostly names of tools (e.g., excel, diary, reminders) and conducted text searches across the entire dataset to identify additional content previously missed. Two authors ( $\mathrm{KC}$ and AS) iteratively coded the data to identify what activities the tools are used for in practice. We conceptually organized activities into broader functions of practice and ran cross-coding comparisons to identify the types of tools used for each function. Any discrepancies were resolved through discussion, and difficult or interesting cases were brought to the larger team for discussion and resolution. We presented initial findings to partners and study participants for comment and reflection.

\section{Results}

We observed that PHIMS played a central role in managing information for program implementation. Although the degree to which practitioners and teams used PHIMS varied, all teams used PHIMS to log information about implementation target achievement for sites, to monitor progress towards overarching scale-up implementation targets, and to keep notes about interactions with site 
contacts. But all teams also used informal tools outside of PHIMS to manage program implementation work and to manage new knowledge about implementation. The following excerpt illustrates how PHIMS is used alongside other tools in practice:

The [practitioner] says I have probably seen how [they] all use their own separate Excel spreadsheet to monitor their sites. To her, "that kind of indicates that really that PHIMS doesn't do everything that we want it to do cause we do keep just a separate [system], I think probably just so it has the most important information that we have so we can just look at it at a glance...without this spreadsheet I would feel a little bit lost with what I'm doing".-Field note from LHD F

\section{Range of informal tools used in addition to PHIMS}

Tools ranged from complex-such as bespoke spreadsheet and database systems designed by the local team-to simple-including standard computer applications and task management tools like email and electronic and physical filing systems. The types of tools observed in practice are described in Table 1. The tools that we observed across most teams were spreadsheets, followed by locally developed templates that practitioners print and take to site visits for notetaking and data collection. Some informal tools existed prior to PHIMS implementation and were adapted over time while others emerged alongside it. The tool used sometimes reflected individual preferences and organization styles and often reflected team approaches to practice. Some teams developed complex spreadsheets or electronically shared files to coordinate work and data entry. Often, multiple tools were used concurrently with PHIMS-a practitioner might have PHIMS opened on one computer screen, email on another, a hardcopy template with notes from a recent site visit, a paper diary, and a spreadsheet tacked to the wall with upcoming deadlines. Informal tools were often used as a complement to PHIMS; for example, spreadsheets were used to manage data downloaded from PHIMS to query specific information. But tools also performed functions that PHIMS was not able to do or could be done more efficiently through another tool. These functions are described in the next section.

\section{Functions of informal tools}

We identified six key functions that informal tools serve in the context of HCI program delivery. These are (1) relationship management, (2) monitoring progress towards target achievement, (3) guiding and troubleshooting PHIMS use, (4) supporting teamwork, (5) conducting an evaluation, and (6) recording work that does not count towards $\mathrm{HCI}$ implementation. A single tool was often used for multiple functions and we observed that all the tools identified were used for many inter-connected functions. These are described in Table 2. In Table 3, we provide excerpts of field notes that are exemplars of the functions that informal tools serve; key themes are highlighted in italics below.

\section{Relationship management}

The most common function of informal tools (observed across 13 teams) was to manage relationships with key

Table 1 Description of (informal) technologies and tools used alongside a standardized monitoring system

\begin{tabular}{|c|c|c|}
\hline $\begin{array}{l}\text { Informal knowledge } \\
\text { management tools }\end{array}$ & Description & $\begin{array}{l}\text { No. of LHDs }(n=14) \text { in } \\
\text { which tool was observed }\end{array}$ \\
\hline Backups, hardcopies from PHIMS & Printing forms from PHIMS to use in hardcopy, or to store in another location & 7 \\
\hline Bespoke IT system/database & Locally designed IT database & 1 \\
\hline Email & Using an email client (e.g., Outlook) externally to PHIMS & 8 \\
\hline \multicolumn{3}{|l|}{ Filing systems } \\
\hline Paper filing system & Storing information in hardcopy in a physical location & 4 \\
\hline Shared folder or drive & Storing information in digital form on a secured, shared drive & 4 \\
\hline Memory & $\begin{array}{l}\text { Instances where memory is explicitly described as a way of capturing and } \\
\text { managing information }\end{array}$ & 3 \\
\hline Online survey tools & Use of online tools (e.g., survey monkey) to gather, manage, or report information & 8 \\
\hline Posted documents & $\begin{array}{l}\text { Information that is in hardcopy and posted close-at-hand, at work stations or } \\
\text { in offices, for quick access }\end{array}$ & 3 \\
\hline Spreadsheet & Spreadsheets/Excel & 12 \\
\hline Task management tools & Systems (e.g., to-do lists, diaries, reminders) to organize and manage tasks & 2 \\
\hline Templates & $\begin{array}{l}\text { Documents that have been locally developed and are standardized to gather } \\
\text { specific types of information }\end{array}$ & 11 \\
\hline Hand written notes and other tools & Not described above; includes handwritten notes & 5 \\
\hline
\end{tabular}




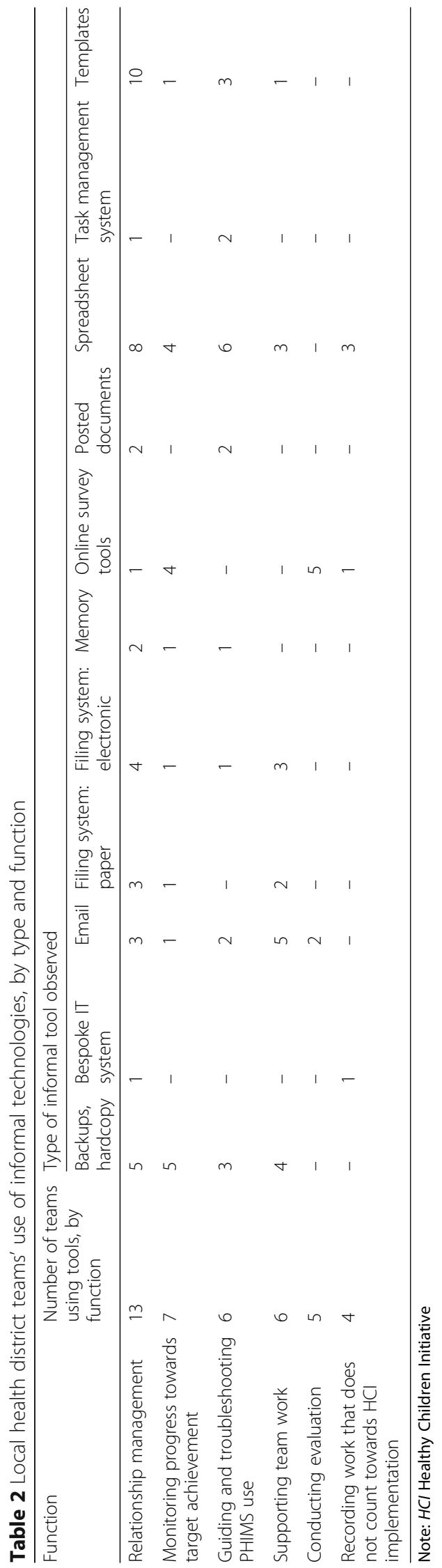




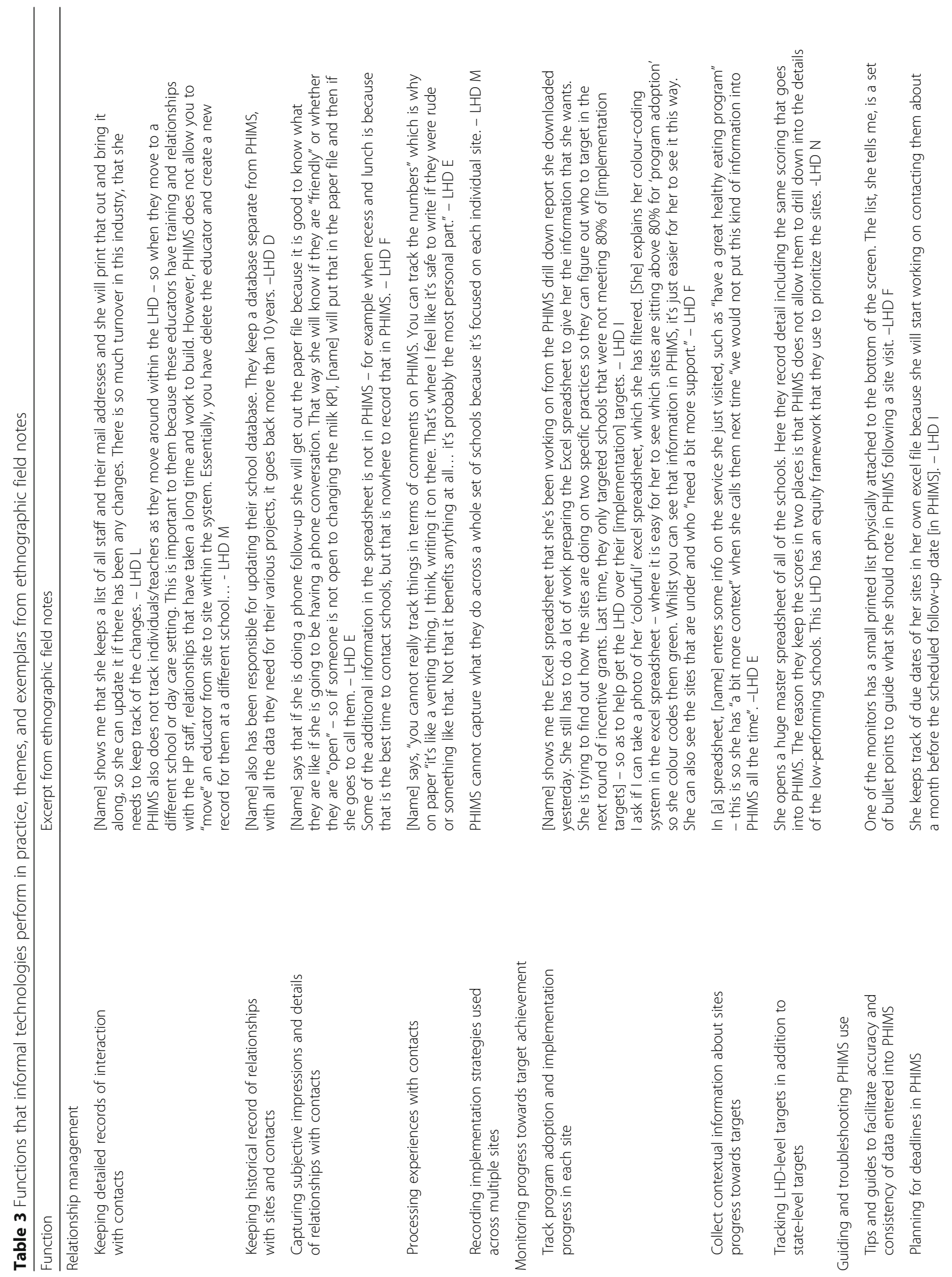




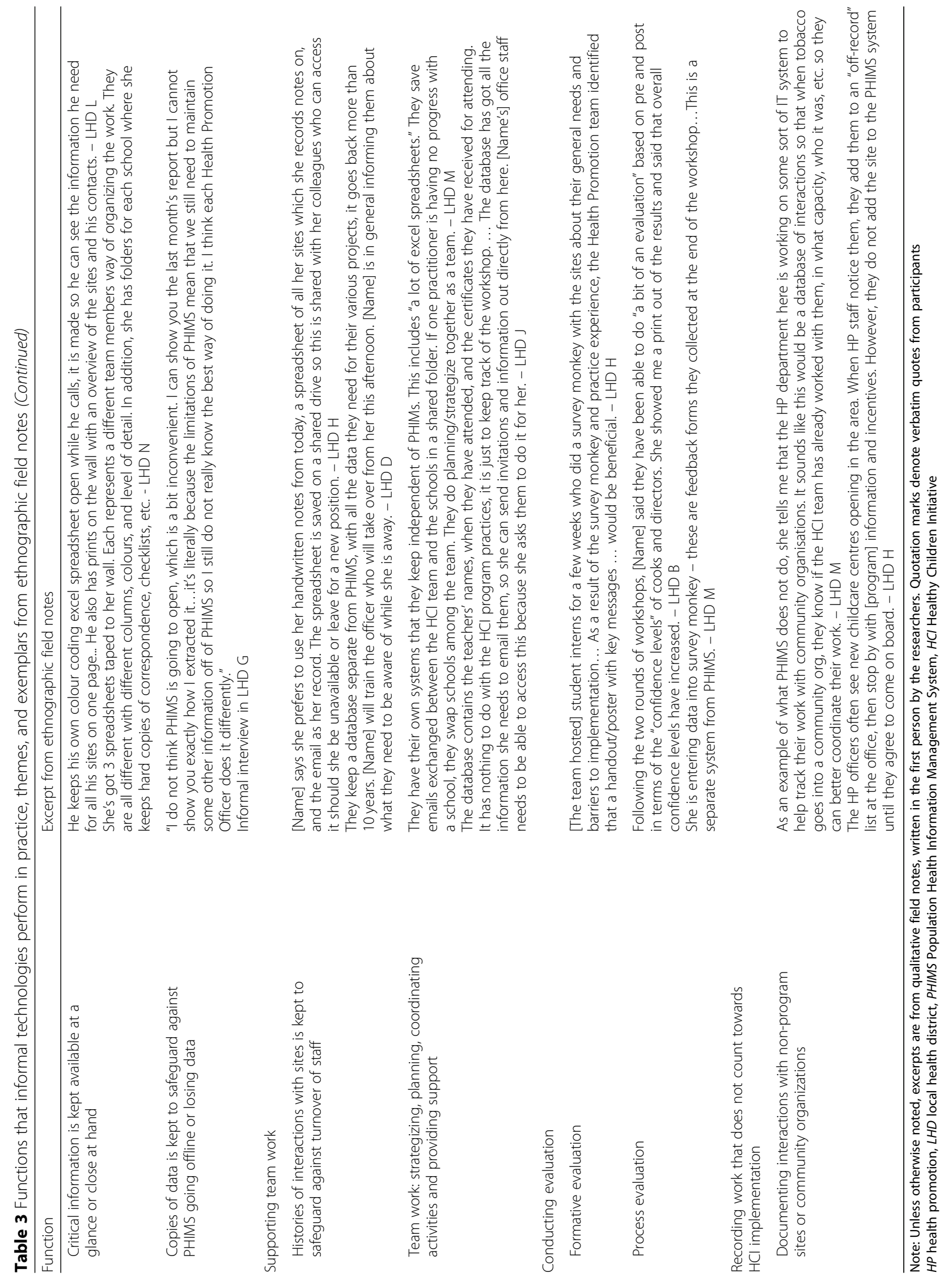


HCI contacts. We observed that all the categories of tools were used to generate and manage knowledge about relationships, including an IT system that had been created by one LHD to manage information about local collaborations. Managing relationships is a key part of implementing HCI programs. PHIMS has inbuilt contact management features for this purpose, but informal tools enabled users to capture more bespoke information. Practitioners used tools outside of PHIMS to keep detailed records of interactions with contacts at sites, including a range of diverse stakeholders. Almost all teams used spreadsheets to track details of sites and stakeholders, record the number of contact attempts they make with a site, and/or to list materials given to contacts. Sometimes, relationships between practitioners, sites, and contacts extended back before the HCI program and PHIMS existed, and informal tools were needed to keep a historical record of relationships with sites and contacts.

Informal tools captured subjective impressions and details of the relationship with contacts. Examples include notes about a stakeholder who is resistant or not ready to make changes to policy and practice, capturing the pet projects of a principal or teacher that could be used to tailor messaging and strategies, or recording the best time of the day to contact a site. Practitioners captured this information via handwritten notes or using templates they developed specifically for this purpose. Informal tools allowed practitioners to write freely, without concern for how their initial impressions might be judged by another in a formal, standardized system like PHIMS. One practitioner explained how writing her initial notes on paper allowed her to process her experience with the contact and "vent" about frustrations experienced before entering a formal note in PHIMS.

Practitioners used informal tools to capture more details about implementation than PHIMS can collect, such as specific information about local contexts. Creating good relationships with site contacts was important to practitioners but can take a long time. Some practitioners wanted to track this work by recording instances of contact and contact attempts. In areas with a high rate of turnover among site staff, practitioners used tools to track staff as they move to new jobs in new sites or to identify new leads. Because PHIMS does not allow practitioners to easily move staff between sites, some LHDs created their own spreadsheets that enabled them to track this information themselves. Similarly, because PHIMS only tracks implementation information at the site level, informal tools capture implementation strategies used across multiple sites, for example, LHD-wide training sessions or the distribution of educational aides that help multiple sites simultaneously progress towards implementation target achievement.

\section{Monitoring progress towards target achievement}

We observed a variety of informal tools used in seven LHDs to monitor sites' progress towards meeting program targets. Informal tools-particularly spreadsheets and templates-were used in conjunction with PHIMS. Practitioners download PHIMS data into spreadsheets to filter for specific information to track implementation progress in each site. This information enabled practitioners to create implementation strategies based on specific criteria, such as sites that are overachieving to serve as case studies or sites that are just under the achievement goal whose improvement could help practitioners meet overall KPI targets.

Practitioners used informal tools to capture contextual information about sites' progress towards meeting targets. Using checklists and templates, practitioners collected detailed information about site-level barriers and facilitators to implementation, e.g., site priorities that might conflict or overlap with efforts to achieve program targets and reminders of contextual information that helps them support sites. Emails were a common tool employed by several LHD teams to document site visits and identify a plan of action, thereby creating a shared record between themselves and the contact for future use.

Informal tools aided collection of additional information about sites that are not required for state-level reporting but is helpful for tracking $L H D$-level targets in addition to state-level targets. One team maintained a spreadsheet that is separate to PHIMS but contains similar and overlapping information. This enabled the team to track their progress against a local equity framework which ensures that teams are attending to schools in high-need areas.

\section{Guiding and troubleshooting PHIMS use}

Bespoke informal tools were created by teams to enable them to use PHIMS better, for instance, by developing a consistent team approach to data entry. Teams developed tip sheets and guides to facilitate the accuracy and consistency of data entered in PHIMS. One participant developed an audit tool to guide and review the qualitative information entered into the system.

The timing of site visits and of data entry is an important component of HCI implementation. KPI data is reported quarterly, there are expectations regarding how often practitioners should meet with sites, and school calendars require practitioners to plan around school holidays when contacts are away. PHIMS has a scheduled reminder function but the users had limited control over when deadlines occur. So practitioners used informal tools to plan for the deadlines that are set in PHIMS, that is, deadlines to report KPI progress or complete a "scheduled follow-up visit" with a site. These tools ranged from simple reminders and 
task lists, to elaborate a spreadsheet that users adapted to their needs. Teams similarly printed hard copies of critical information from PHIMS and other electronic sources to keep at hand for critical information needed "at a glance". Spreadsheets, checklists, and information sheets were observed hanging in many offices and cubicles containing information about timelines, priority lists, and reminders about when to follow up with key contacts.

Tools are used by teams to create backups of important data to safeguard against PHIMS going offline or losing data. This concern is based on known problems with PHIMS, namely periods of planned or unexpected server outages and difficulties in finding or "losing" data in PHIMS. Keeping backups reflected a mistrust of the formal, standardized system, including confusion about how it works, where data goes, and anxiety around losing data if changes are made. However, keeping data in multiple places also posed problems for ensuring data quality. One practitioner discussed potential "measurement error" due to mistakes in transposing data across multiple systems.

I ask [Name removed] if she has other systems to record data, and she tells me, "[name] is a spreadsheet queen, that's my gripe with her. Everything's recorded in about 7 different places [this is said as a platitude]. Which is irritating because to me I just see measurement error. Like, screaming at me because sometimes you forget to put it there or you miss it there and then it gets transferred or someone saves it incorrectly. I think it stems because PHIMS came in a few years ago and had a lot of teething problems and they kept missing or losing data." - Field note from LHD E

\section{Supporting teamwork}

Using informal tools to create detailed histories of work with sites alleviated another concern: transferring knowledge to future practitioners in the case of potential turnover so that knowledge about a site does not reside with only one practitioner. Teams also use other tools to strategize together, create plans, and coordinate activities. Having a variety of records available to the whole team via electronic or physical filing systems enabled practitioners to create knowledge together by sharing resources and information about sites with one another and by collaborating to share and create strategies to address barriers.

\section{Conducting evaluation}

Conducting evaluation is a key competency in health promotion but not required of HCI staff at the local level. However, many practitioners performed evaluation activities to develop knowledge to help guide their approach to $\mathrm{HCI}$. Informal tools, primarily online survey tools, were used to conduct formative evaluations to gain new insights about needs across sites. This information informed the design of educational aides, training, and other activities to support sites and increase KPIs. Practitioners sometimes entered evaluation data from workshops and events into online survey tools which enable them to easily analyze, tabulate, and report results.

\section{Recording work that does not count towards $\mathrm{HCl}$ implementation}

The final function we identified is that informal tools capture work that is outside the scope of HCI implementation. Teams used informal tools to document their interaction with non-HCI sites and community organizations. One team created a spreadsheet to collect information about new childcare businesses opening in the area. This team would work to support these sites but only enroll them in PHIMS after they agreed to participate in the formal program.

\section{Discussion}

By examining PHIMS use in practice and the informal tools that arose alongside, we demonstrate the kind of information that practitioners value for implementation. While we observed that practitioners valued information to help them achieve HCI goals, we also observed that they valued information reflective of broader competencies and values of health promotion practice [28]-e.g., evaluation, teamwork, succession planning, innovation, respectful documentation of practice, and partnership building. Informal tools, therefore, served a range of important practice functions that enhanced both efficiency and flexibility of HCI work and provided functions not accommodated by PHIMS. These informal systems largely did not displace PHIMS; rather, they appeared to be complementary by enabling teamwork and integration of PHIMS into practice routines.

\section{Knowledge for implementation}

We found practitioners and teams drew from a variety of tools and technologies outside of a standardized IT information management system to implement $\mathrm{HCI}$. While in some instances, informal tools served as "workarounds" to limitations of PHIMS, their use went beyond that. By drawing on informal tools, practitioners generated information about trends and patterns of implementation across sites. Informal tools enabled practitioners to generate new knowledge about effective and efficient contextspecific strategies to meet implementation targets. This finding reflects previous studies that illustrate that users who can access multiple types of technology use them congruently and concurrently to aide in practice and production of knowledge [29]. This is because most learning in 
organizational settings is informal, and thereby, facilitated by access to a variety of informal technologies [30,31].

Informal tools provide a low-risk and low-investment option for experimenting with knowledge creation, whereas even simple design changes to standardized IT systems can be costly. In our study, practitioners used information recorded in informal tools to reflect on and make sense of their practice experiences, creating new knowledge about relationships and implementation that were eventually translated into data for the standardized PHIMS system. Templates and handwritten notes provided an efficient way to capture impressions that might change over the course of single or subsequent interactions or to track emergent barriers that may or may not prove influential to implementation. If the information captured via these systems turns out to be irrelevant, it can be easily changed or discarded. Conversely, information entered into standardized systems becomes permanently codified in the system and may be difficult to amend should impressions prove inaccurate or information proves meaningless [32]. The imperative of delineating types of knowledge and how to capture it is particularly relevant in contexts where personal and professional realms are blurred. Informal tools enabled practitioners to straddle the line between personal and professional. For instance, practitioners used handwritten notes to create memory aids for future interactions, while simultaneously maintaining a degree of privacy and discretion over the information they chose to input into PHIMS.

\section{Implementation and design of PHIMS}

Some may argue that the use of informal tools alongside PHIMS could indicate that PHIMS is not fulfilling its intended purpose well enough. This position reflects an implementation science perspective in which the process of scale and spread is conceptualized as sequential and structured. In this perspective, IT systems enable standardization and replication of core components across sites. But our findings are better interpreted through a complexity lens [33]. Complexity theory posits that complex systems (made up of things, people, and process) are dynamic-constantly adapting in response to changes in context. That practitioners are using alternate systems with, and sometimes instead of, PHIMS is an example of users adapting and modifying technologies in sometimes unexpected, but retrospectively understandable, ways.

From its inception, the vision for PHIMS was a system that could support teams in coordinating implementation in addition to data collection for monitoring and reporting purposes. The designers adopted user-centered design principles and undertook a lengthy consultation process. However, this process was undertaken almost 5 years ago. It is important to note that PHIMS is "living" and has been adapted and updated. But this study provides deeper insights that may signal the extent to which the broader system of $\mathrm{HCI}$ implementation, including what the program is and what it takes to implement it, has changed over time. The functions we identify invite reflection on how the vision for PHIMS might also adapt to better support the increasingly sophisticated knowledge being developed by practitioners about $\mathrm{HCI}$ implementation. There are current existing mechanisms that could be strengthened to improve user feedback to inform adaptations to both PHIMS and its broader operating environment, i.e., in what ways it is used to support HCI. In addition, the results of this partnership research project are contributing insights for PHIMS system improvement (Conte, KP and Davidson, S. Using a rich picture' to facilitate systems thinking in research coproduction. In submission). Fully embracing the view that IT system development is an ongoing, iterative process that persists after initial design and implementation will be critical to ensuring its ongoing relevance and sustainability [34]. While recording systems have traditionally been used to track standardized key components of practice [9], computer technologies are becoming more responsive and better supporting userdefined adaptations. As electronic monitoring systems continue to develop, there is potential to design them to better capture emerging knowledge and innovations.

The use of informal systems also invites reflection on the appropriate delineation of the information that needs to be standardized and codified via an institutional IT system versus the type of information that should remain for local use only. It is likely infeasible to legitimately standardize implementation processes within a formal IT system across such a large geographical area with very different organizational arrangements and numbers of sites. Local nuances and variations in implementation approaches pose meaningful differences in practice that could be restrained through attempts at standardization. Further, formal largescale IT systems like PHIMS have multiple users whose conflicting work processes often collide via the technology. For example, the value of seemingly tedious data entry may be unclear to users on the front lines of practice but may be justifiable to administrators responsible for the overall delivery and ensuring future funding $[35,36]$. These issues are an invitation to resist over-standardization and to recognize the infeasibility of formal systems to fully anticipate and meet the needs of all possible users. Shifting contexts and information needs will require nimbleness, and informal systems allow for that. Formal IT systems may only need to serve a few key purposes well (e.g., monitoring and/or knowledge sharing) while allowing secondary functions to be added depending on need and purpose.

\section{Limitations}

It is likely that we were unable to capture the full range and instances of informal tools and systems that were used alongside PHIMS in practice. Ethnography is an 
opportunistic method which means that the data captured is dependent on what researchers have an opportunity to see or are allowed to see. The degree to which we obtained access varied across sites and precluded an in-depth analysis of contextual factors that might explain or predict the adoption of informal tools. We were less interested in documenting the prevalence of informal systems in practice, instead of exploring what it means that they are used at all and for what purposes. We are unable to fully appreciate whether the use of informal tools constitutes a threat to PHIMS use and sustainability. Future questions to ask are perhaps are informal tools a problem; whose problem are they; and if they are not a problem now, when might they become one? Our responsibility is to promote the dialog to address these issues. Another interpretation is that informal systems may support formal systems and thus help explain why PHIMS has been sustained while other similar systems have been abandoned [37, 38]. PHIMS is, in essence, a process or bundle of activities. The informal tools reflect practitioners' dedication to practice, a value-add.

\section{Conclusion}

Based on our observations, we conclude that PHIMS is serving important practice functions: the coordination, standardized reporting, and tracking of the large-scale implementation of a prevention program-something that, to our knowledge, has never been done at a large scale in population-level prevention. PHIMS may be doing less well at fulfilling its purpose as a practice-support system than intended, but it may be that doing well enough is the key to its success. We have also shown that while implementers use PHIMS to aide implementation, they also use informal tools to generate new knowledge that supports program delivery and quality practice. This knowledge complements standardized monitoring systems by enabling knowledge generation about implementation while supporting the integration of standardized systems to monitor KPIs. The challenge is to strike the right balance between the two: allowing for flexibility without overstandardizing practice.

\section{Supplementary information}

Supplementary information accompanies this paper at https://doi.org/10. 1186/s13012-019-0938-7.

Additional file 1. Additional information as per the consolidated criteria for reporting qualitative research (COREQ) checklist.

\section{Abbreviations}

HCl: Healthy Children Initiative; IT: Information technology; KPIs: Key performance indicators; LHDs: Local health districts; NSW: New South Wales: PHIMS: The Population Health Information Management System

\section{Acknowledgements}

We thank colleagues in the NSW Ministry of Health and local health districts for their input and agreement to develop this project and their ongoing participation.

\section{Authors' contributions}

KC conducted the final analysis for this paper and drafted the manuscript. AS conducted the initial analysis and contributed to early drafting guided by $\mathrm{KC}$ and PH. KC, VL, and SG participated in the collection and analysis of data. PH and KC developed the protocol and study approach. PH conceptualized the study in response to critical reflection on PHIMS' development by ST and JM. $A G, A M, S T, L P, C l, J M$, and MW participated in the design of the study. All authors made important contributions to the theoretical approach and interpreting insights. All authors read, revised, and approved the final manuscript.

\section{Funding}

This work is funded by the National Health and Medical Research Council of Australia (NHMRC) through its partnership center grant scheme (Grant ID: GNT9100001). NSW Health, ACT Health, The Commonwealth Department of Health, The Hospitals Contribution Fund of Australia, and HCF Research Foundation have contributed funds to support this work as part of the NHMRC partnership center grant scheme.

\section{Availability of data and materials}

The datasets generated and/or analyzed during the current study are not publicly available due information that could compromise research participant consent and privacy but can be made available from the guaranteeing author $(\mathrm{PH})$ subject to appropriate precautions and upon reasonable request.

\section{Ethics approval and consent to participate}

Research ethics approval has been granted by the Royal Prince Alfred Hospital Human Research Ethics Committee (X16-0156) and by the research governance offices of each of the LHDs.

\section{Consent for publication}

Not applicable.

\section{Competing interests}

The NSW Ministry of Health is one of five funding partners of The Australian Prevention Partnership Centre (TAPPC), within which this project is situated. AM, LP, ST, and JM are employees of the NSW Ministry of Health which funds and monitors the performance of the Healthy Children's Initiative $(\mathrm{HCl})$, on which program this research is focused and the participants are employed. AG, $\mathrm{ClH}$ are employees of the Office of Preventive Health which is responsible for supporting the statewide implementation of $\mathrm{HCl}$. MW oversees the health promotion unit at one of the study sites. LP, JM, MW, and ST are part of a team that designed, manages, and maintains the PHIMS system described in this paper. KC, VL, SG, and PH are university-based researchers funded through TAPPC to conduct this research. To ensure confidentiality of participants, identifying data about the individuals and the local health districts in which they work was only available to the university-based researchers. Only illustrative, de-identified quotes were shared with the research partners in the context of developing papers.

\section{Author details}

${ }^{1}$ The Australian Prevention Partnership Centre, Ultimo, NSW 2007, Australia. ${ }^{2}$ Menzies Centre for Health Policy, School of Public Health, and University Centre for Rural Health, Faculty of Medicine and Health, The University of Sydney, Sydney, New South Wales 2006, Australia. ${ }^{3}$ McMaster University, Hamilton, Ontario, Canada. ${ }^{4}$ The Australian Prevention Partnership Centre, based at the Menzies Centre for Health Policy, School of Public Health, Faculty of Medicine and Health, The University of Sydney, Sydney, New South Wales, Australia. ${ }^{5} \mathrm{New}$ South Wales Office of Preventive Health, New South Wales Ministry of Health, Sydney, Australia. ${ }^{6} \mathrm{Centre}$ for Epidemiology and Evidence, New South Wales Ministry of Health, Sydney, New South Wales, Australia. ${ }^{7}$ Health Promotion Service, South Western Sydney Local Health District, Liverpool, New South Wales, Australia. ${ }^{8}$ Centre for Population Health, New South Wales Ministry of Health, Sydney, New South Wales, Australia. 
Received: 19 March 2019 Accepted: 4 September 2019

Published online: 18 September 2019

\section{References}

1. Rissel C, Laws R, St George A, Hector D, Milat AJ, Baur LA. Research to practice: application of an evidence-building framework to a childhood obesity prevention initiative in New South Wales. Health Promot J Austr. 2012;23(1):16-24.

2. World Health Organization. Report of the commission on ending childhood obesity. Geneva: World Health Organization; 2016.

3. Ipsos-Eureka. Evaluation of the Live Life Well @ School initiative: final report. Sydney: New South Wales Department of Health; 2010.

4. Hardy LL, King L, Kelly B, Farrell L, Howlett S. Munch and Move: evaluation of a preschool healthy eating and movement skill program. Int J Behav Nutr Phys Act. 2010;7(1):1-11.

5. Innes-Hughes $C$, Rissel C, Thomas M, Wolfenden L. Reflections on the NSW Healthy Children Initiative: a comprehensive state-delivered childhood obesity prevention initiative. Public Health Res Pract. 2019;29(1):e2911908.

6. Lockeridge A, Innes-Hughes C, O'Hara BJ, McGill B, Rissel C. Munch and Move: evidence and evaluation summary. North Sydney: NSW Ministry of Health; 2015.

7. Bravo A, Innes-Hughes C, O'Hara BJ, McGill B, Rissel C. Live Life Well @ School: evidence and evaluation summary 2008-2015. North Sydney: NSW Ministry of Health; 2016.

8. Procter R, Wherton J, Greenhalgh T. Hidden work and the challenges of scalability and sustainability in ambulatory assisted living. Acm Trans Comput Hum Interact. 2018;25(2):11.

9. Conte KP, Hawe P. Will E-monitoring of policy and program implementation stifle or enhance practice? How would we know? Front Public Health. 2018; 6:243.

10. New South Wales Ministry of Health. Service Agreement Template. Sydney: New South Wales Ministry of Health; 2016.

11. Farrell L, Lloyd B, Matthews R, Bravo A, Wiggers J, Rissel C. Applying a performance monitoring framework to increase reach and adoption of children's healthy eating and physical activity programs. Public Health Res Pract. 2014;25(1):e2511408.

12. Green A, Innes-Hughes C, Rissel C, Mitchell J, Milat A, Williams M, et al. Codesign of the Population Health Information Management System to measure reach and practice change of childhood obesity programs. Public Health Res Pract. 2018;28(3):e2831822.

13. Conte KP, Groen S, Loblay V, Green A, Milat A, Persson L, et al. Dynamics behind the scale up of evidence-based obesity prevention: protocol for a multi-site case study of an electronic implementation monitoring system in health promotion practice. Implement Sci. 2017;12(1):146.

14. Tong A, Sainsbury P, Craig J. Consolidated criteria for reporting qualitative research (COREQ): a 32-item checklist for interviews and focus groups. Int Qual Health Care. 2007;19(6):349-57.

15. O'Brien BC, Harris IB, Beckman TJ, Reed DA, Cook DA. Standards for reporting qualitative research: a synthesis of recommendations. Acad Med. 2014:89(9):1245-51.

16. Marcus GE. Ethnography in/of the world system: the emergence of multisited ethnography. Annu Rev Anthropol. 1995;24:95-117.

17. Burrell J. The field site as a network: a strategy for locating ethnographic research. Field Methods. 2009:21(2):181-99.

18. Fortun K. Scaling and visualizing multi-sited ethnography. In: Falzon M, editor. Multi-sited ethnography. London: Routledge; 2009.

19. Knoblauch H. Focused ethnography. Forum Qualitative Sozialforschung / Forum: Qualitative Social Research. 2005;6(3).

20. Blumer H. Symbolic interactionism: perspective and method. Berkeley: Univ of California Press; 1986

21. Pink S, Morgan J. Short-term ethnography: intense routes to knowing. Symb Interact. 2013;36(3):351-61.

22. Vindrola-Padros C, Vindrola-Padros B. Quick and dirty? A systematic review of the use of rapid ethnographies in healthcare organisation and delivery. BMJ Qual Saf. 2018:27(4):321-30.

23. Boyer D. Reflexivity reloaded: from anthropology of intellectuals to critique of method to studying sideways; 2014. p. 91-110.

24. Holmes D, Marcus GE. Fast-capitalism: para-ethnography and the rise of the symbolic analyst. Frontiers of capital: ethnographic reflections on the new economy. Durham: Duke University Press; 2006. p. 33-57.
25. Vindrola-Padros C, Pape T, Utley M, Fulop NJ. The role of embedded research in quality improvement: a narrative review. BMJ Qual Saf. 2017:26(1):70.

26. Hsieh HF, Shannon SE. Three approaches to qualitative content analysis. Qual Health Res. 2005;15(9):1277-88.

27. Nvivo qualitative data analysis software. 11 ed: QSR International Pty Ltd.; 2015.

28. Australian Health Promotion Association. Core competencies for health promotion practitioners. Maroochydore: Australian Health Promotion Association; 2009. https://www.healthpromotion.org.au/images/docs/core_ competencies_for_hp_practitioners.pdf.

29. Jarrahi MH, Sawyer S. Social technologies, informal knowledge practices, and the enterprise. J Organ Comput Electron Commer. 2013;23(1-2):110-37.

30. Caruso SJ. A foundation for understanding knowledge sharing: organizational culture, informal workplace learning, performance support, and knowledge management. Contemp Issues Educ Res. 2017;10(1):45.

31. Davison RM, Ou CXJ, Martinsons MG. Information technology to support informal knowledge sharing. Inf Syst J. 2013;23(1):89-109.

32. Flanagan ME, Saleem JJ, Millitello LG, Russ AL, Doebbeling BN. Paper- and computer-based workarounds to electronic health record use at three benchmark institutions. J Am Med Inform Assoc. 2013;20(e1):e59-66.

33. Greenhalgh T, Papoutsi C. Spreading and scaling up innovation and improvement. BMJ. 2019;365:12068.

34. Cresswell KM, Bates DW, Sheikh A. Ten key considerations for the successful optimization of large-scale health information technology. J Am Med Inform Assoc. 2017:24(1):182-7.

35. Greenhalgh T, Potts HWW, Wong G, Bark P, Swinglehurst D. Tensions and paradoxes in electronic patient record research: a systematic literature review using the meta-narrative method. Milbank Q. 2009;87(4):729-88.

36. Symon G, Long K, Ellis J. The coordination of work activities: cooperation and conflict in a hospital context. Comput Supported Coop Work. 1996;5(1):1-31.

37. Bors PA, Kemner A, Fulton J, Stachecki J, Brennan LK. HKHC community dashboard: design, development, and function of a web-based performance monitoring system. J Public Health Manag Pract. 2015; 21(Suppl. 3):S36-44.

38. Thomas C, Smith B, Wright-DeAguero L. The program evaluation and monitoring system: a key source of data for monitoring evidence-based HIV prevention program processes and outcomes. AIDS Educ Prev. 2006;18(4 Suppl A):74-80.

\section{Publisher's Note}

Springer Nature remains neutral with regard to jurisdictional claims in published maps and institutional affiliations.

Ready to submit your research? Choose BMC and benefit from:

- fast, convenient online submission

- thorough peer review by experienced researchers in your field

- rapid publication on acceptance

- support for research data, including large and complex data types

- gold Open Access which fosters wider collaboration and increased citations

- maximum visibility for your research: over $100 \mathrm{M}$ website views per year

At BMC, research is always in progress.

Learn more biomedcentral.com/submissions 